\title{
GalNAc-siRNA Conjugates: Leading the Way for Delivery of RNAi Therapeutics
}

\author{
Aaron D. Springer and Steven F. Dowdy
}

\begin{abstract}
Short-interfering RNA (siRNA)-induced RNAi responses have great potential to treat a wide variety of human diseases from cancer to pandemic viral outbreaks to Parkinson's Disease. However, before siRNAs can become drugs, they must overcome a billion years of evolutionary defenses designed to keep invading RNAs on the outside cells from getting to the inside of cells. Not surprisingly, significant effort has been placed in developing a wide array of delivery technologies. Foremost of these has been the development of $N$-acetylgalactosamine (GalNAc) siRNA conjugates for delivery to liver. Tris-GalNAc binds to the Asialoglycoprotein receptor that is highly expressed on hepatocytes resulting in rapid endocytosis. While the exact mechanism of escape across the endosomal lipid bilayer membrane remains unknown, sufficient amounts of siRNAs enter the cytoplasm to induce robust, target selective RNAi responses in vivo. Multiple GalNAc-siRNA conjugate clinical trials, including two phase III trials, are currently underway by three biotech companies to treat a wide variety of diseases. GalNAc-siRNA conjugates are a simple solution to the siRNA delivery problem for liver hepatocytes and have shown the RNAi (and antisense oligonucleotide) field the path forward for targeting other tissue types.
\end{abstract}

Keywords: $N$-acetylgalactosamine, clinical trials, liver

\section{Introduction}

$S^{\mathrm{n}}$ HORT-INTERFERING RNA (SIRNA)-induced RNAi responses have great potential to treat a wide variety of human diseases from cancer to pandemic viral outbreaks to Parkinson's Disease [1-3]. Moreover, siRNAs have the potential to pharmaco-evolve their targeting sequence to keep pace with mutations in diseases driven by genetic change, such as cancer and influenza, a property that no other clinical modality can perform. However, before siRNAs can become drugs, they must overcome a billion years of evolutionary defenses designed to keep invading RNAs on the outside cells from getting to the inside of cells [1-3]. Consequently, since the beginning of the RNAi therapeutic revolution, the problem to solve has remained the same: Delivery! Delivery! Delivery! Indeed, all other issues with developing siRNAs as a therapeutic modality have paled in comparison to the delivery problem. Not surprisingly, there has been significant attention and investment of time and resources to solve the delivery problem by harnessing and developing a wide array of technologies [1-3].

Early approaches focused on lipid nanoparticles (LNPs) and synthetic nanoparticles to address the siRNA delivery problem that were predicated on several decades worth of nanoparticle delivery approaches developed for large DNA gene therapy vectors $[2,4]$. LNPs serve to mask the siRNA charge, protect it from degradation by RNases, and facilitate endosomal escape into the cytoplasm. LNPs also allowed for the use of minimally modified siRNA backbones that primarily contained native 2 '-hydroxyl groups. However, native double-stranded siRNAs are recognized as invading nucleic acids by multiple cellular defense mechanisms, including extracellular toll-like receptors (TLR-3, -7, -8) and intracellular sensors retinoic acid inducible gene (RIG-I) and melanoma differentiation associated protein 5 (MDA-5) [5]. Fortunately, incorporation of Fluoro (F) and Methoxy (OMe) modifications into the $2^{\prime}$ position of the ribose ring dramatically reduces both of these activities and, importantly, is highly tolerated by Ago [1-3,6]. Because 2 '-modifications greatly increase the siRNA stability and overall half-life, that opened the door for conjugating targeting domains directly to naked siRNAs and thereby avoiding the use of LNPs, which are some 5,000-fold larger than the siRNA drug being delivered.

The prototypical siRNA conjugate is a trimer of $\mathrm{N}$ acetylgalactosamine (GalNAc), which avidly binds to the Asialoglycoprotein receptor (ASGPR) that is predominantly expressed on liver hepatocytes [7,8]. GalNAc-siRNA conjugates have been thoroughly investigated in preclinical rodent and nonhuman-primate (NHP) models and are currently being tested in multiple clinical trials sponsored by three biotech companies. None of this would have been possible if it were not for the convergence of 50+ years of prior work on

Department of Cellular and Molecular Medicine, University of California San Diego, La Jolla, California. 
ASGPR with $50+$ years of nucleic acid chemistry. GalNAcsiRNA conjugates serve as a simple solution to the delivery problem for liver hepatocytes and have shown the RNAi (and antisense oligonucleotide [ASO]) field the path forward for targeting other tissue types.

\section{Identification of the ASGPR}

ASGPR, also known as hepatic binding protein or the Ashwell-Morell receptor, is the first animal lectin to be detected $[9,10]$. ASGPR was first "accidently" discovered as early as 1965 by Gilbert Ashwell and Anatol Morell in the course of studying a circulating glycoprotein, ceruloplasmin, in rabbits [11]. An attempt to determine ceruloplasmin's circulating half-life by removing terminal sialic acids and radiolabeling the resulting terminal galactose led instead to the observation that asialoceruloplasmin rapidly disappeared from serum and was fully recoverable in the liver within 5-10 min [12,13].

By 1968, Ashwell and Morell had determined that galactose was the necessary terminal sugar residue for binding to the yet unnamed ASGPR. Removal or oxidation of the terminal galactose by $\beta$-galactosidase or galactose oxidase, respectively, inhibited clearance of the labeled asialoceruloplasmin. Localization was observed specifically in hepatocytes with total exclusion from Kupffer cells, suggesting that clearance of asialoceruloplasmin is unique from heat killed controls and indicating a specific mechanism [7,13]. By the early 1970s, ASGPR activity was isolated to membrane fractions of rat and rabbit liver and determined to be $\mathrm{pH}$ sensitive with an absolute requirement for $\mathrm{Ca} 2+$ to maintain binding activity $[14,15]$. Blood type specific agglutination confirmed ASGPR as a lectin and demonstrated for the first time that the affinity for GalNAc was higher than galactose [16,17].

In 1970, partial replacement of sialic acid residues on asialoglycoproteins showed a binding threshold for galactose residues, indicating that at least two galactose residues were required for rapid clearance of asialoglycoproteins [18,19]. In addition, preference in clearance among various co-injected asialoglycoproteins and peptides suggested that both the galactose residue number and arrangement play a role in ligand binding $[7,20]$. These early studies went on to show that binding depended on the type of sugar (GalNAc $>$ Gal) and number of sugars with $4=3>2>1$ [21-24]. Recent studies showed that geometrical spacing between the sugars is also important [25]. X-ray crystal structures of the extracellular domain of ASGPR revealed a shallow carbohydrate binding pocket, explaining the requirement for multivalency [26,27].

GalNAc binding to ASGPR occurs at the sinusoidal surface of the hepatocyte [14-28], which contains $\sim 500,000$ receptors per cell $[10,22,29,30]$, of which about $5 \%-10 \%$ are present at the cell surface at any one time [22,31]. GalNAc binding initiates on diffuse monomeric ASGPR receptors, followed by rapid local aggregation of ligand bound receptors leading to larger scale aggregation in clathrin coated pits, proceeding to endocytosis [22,32-35]. Early studies noted that the ASGPR half-life was much longer than the bound asialoglycoproteins [36]. Subsequent studies showed that acidification during endosomal maturation leads to dissociation of the GalNAc ligand from ASGPR followed by GalNAc degradation in the lysosome [37] and recycling of ASGPR to the cell surface (Fig. 1) [38-40].

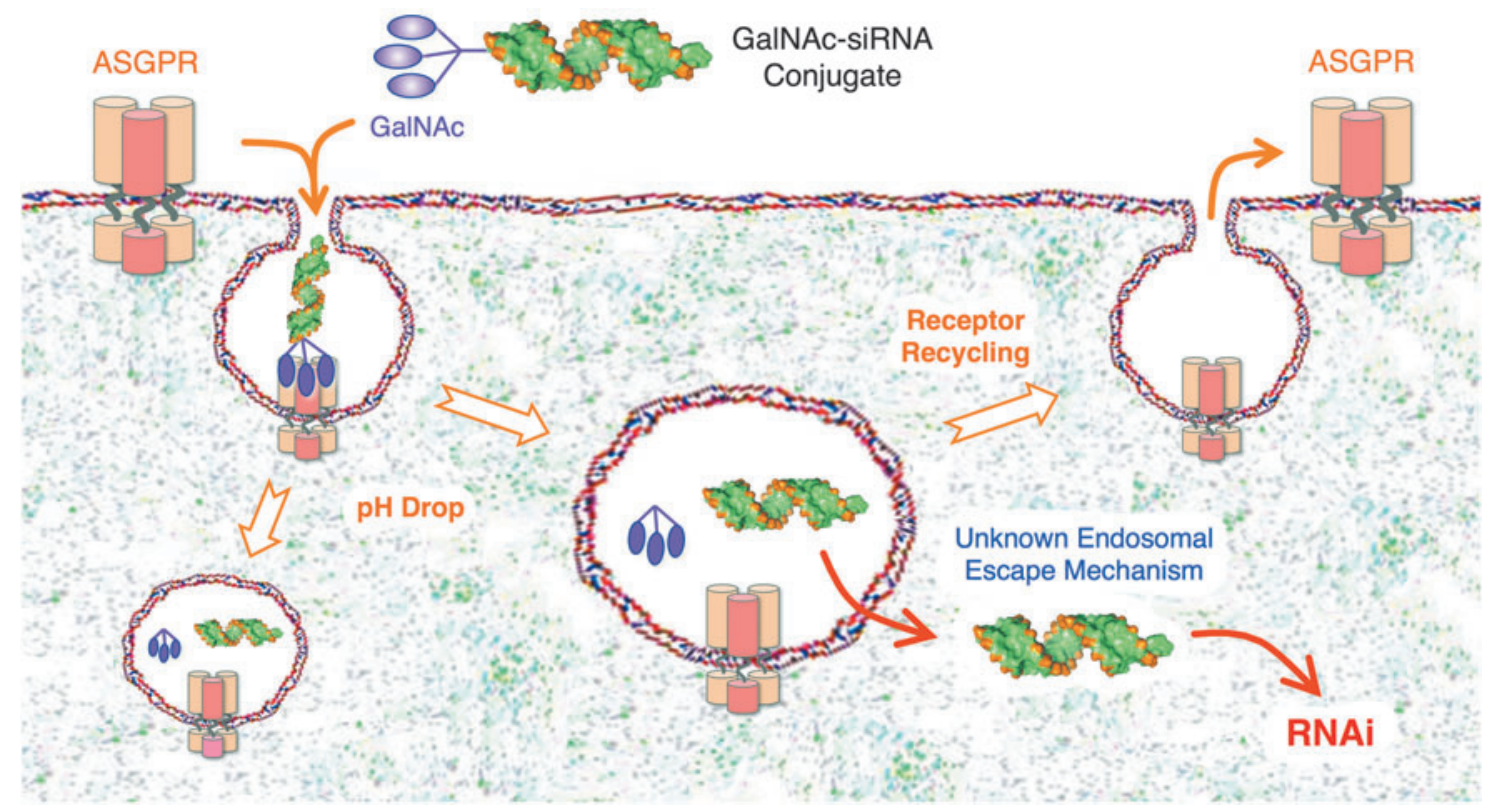

FIG. 1. Delivery of GalNAc-siRNA conjugates into hepatocytes. Approximately $10^{6}$ ASGPR resides on the surface of liver hepatocytes, and upon binding, sialyl-GalNAc molecules are rapidly engulfed into hepatocytes by endocytosis. Due to a $\mathrm{pH}$ drop, GalNAc-siRNA conjugates are released from ASGPR into the lumen of the endosome, and ASGPR recycles back to the hepatocyte surface. GalNAc and the linkers are rapidly degraded off of the siRNA conjugate and by a currently unknown mechanism, a small fraction of free siRNA, likely $<1 \%$, escapes across the endosomal lipid bilayer membrane into the cytoplasm of the hepatocyte. Once in the cytoplasm, siRNAs are rapidly loaded by transactivation responsive RNAbinding protein into Ago to induce robust and sustained RNAi responses. GalNAc, $N$-acetylgalactosamine; ASGPR, asialoglycoprotein receptor; siRNA, short-interfering RNA. 


\section{Early Work on GalNAc-Nucleic Acid Conjugates Targeting the Liver}

The clinical possibilities of ASGPR were realized as early as 1971 when delivery of nonglycoproteins to the liver was accomplished through conjugation to asialofetuin [41]. Liver targeting of nonglycoproteins through conjugation to lactose in 1978 demonstrated that alteration of proteins with targeting galactose residues was sufficient for delivery [42]. The first targeted delivery of a biologically active molecule to hepatocytes in vivo was demonstrated in 1979 when injection of asialofetuin-linked trifluorothymidine reduced hepatic Ectromelia viral DNA replication threefold in rats [43]. Delivery of additional antivirals [44], low-density lipoprotein (LDL) [45], and diphtheria toxin [46] demonstrated that targeting of ASGPR is capable of delivering diverse cargo and eliciting an array of biological responses in vivo. In the following decades, asialoglycoproteins, galactose, and galactose derivatives, including GalNac, were widely investigated to deliver biologically active glycopeptides [24], glycolipids [47], small molecules [48,49], nucleoside analogs [43,44,49,50], plasmid DNA [51-55], and ASOs [56-60] to liver hepatocytes.

Throughout the 1980s and 1990s, plasmid DNA was delivered using polylysine complexes to form nanoparticles. Early work in the late 1980s showed that delivery utilizing asialo-orosomucoid-poly-lysine conjugates targeted plasmid DNA to hepatocytes in vitro $[51,53]$. The first in vivo delivery utilizing this asialo-orosomucoid-poly-lysine approach showed rapid and selective expression of plasmid encoded protein in the hepatocytes $24 \mathrm{~h}$ postintravenous injection [52,61]. By the early 1990s, structurally defined DNA-binding ligand conjugates were created using multivalent galactose and GalNAc. This reduced the complexity and size of the previous asialoorosomucoid conjugate nanoparticles and effectively delivered DNA to hepatocytes in vivo $[54,55]$. Despite these advances, plasmid transfection efficiency was very low, with over $50 \%$ of the DNA degraded in the lysosome [62]. Co-injection with defective viral particles increased plasmid DNA delivery 500fold [54], highlighting the importance of endosomal escape in ASGPR-mediated delivery, a rate-limiting issue that persists today.

\section{Improving the Chemical Design of siRNAs}

In 2001, following up on the 1998 Nobel prize winning work of Fire and Mello on RNAi in Caenorhabditis elegans [63], work performed in Tuschl's laboratory [64] demonstrated the ability of synthetic siRNAs to modulate protein expression in mammalian cells, including human cells. siRNA therapeutics offer the promise of targeting previously undruggable disease genes, including transcription factors and intractable oncogenes, such as MYC and KRAS. Most impressively, the siRNA's targeting sequence can be pharmaco-evolved to target new mutations that appear in recurrent cancer and influenza, thereby allowing the siRNA drug to keep pace with the mutational rate [1].

Unlike ASOs, siRNAs remain inactive inside the cell until loaded by transactivation responsive RNA-binding protein (TRBP) into Argonaute (Ago2) where the passenger (sense) strand is removed, leaving the guide (antisense) strand bound to catalytic Ago2 [65]. Ago2 provides guide strand structural context for the initial binding of the guide strand seed region to the target mRNA [66,67], thereby dramatically lowering the activation energy compared to a free antisense strand oligonucleotide [68].

Despite RNAi's promising therapeutic features, due to its size $(\sim 14,000 \mathrm{Da})$ and 40 negatively charged phosphates, siRNA RNAi therapeutics cannot enter cells on their own and require a delivery agent [1-3]. Moreover, naked siRNAs are rapidly degraded in blood by RNAses, cleared by the kidneys, absorbed by liver scavenger receptors, and can activate the innate immune TLR3/7/8, RIG-I, and MDA-5 systems [1$3,5,69,70]$. All these factors contribute to a poor drug profile for siRNA, which must be addressed before the therapeutic potential of siRNAs can be realized.

Traditional small molecule chemistry, manufacturing and controls (CMC) optimization strategies fail to fully address these limitations as siRNAs have several biological requirements for activity. First, TRBP contains three double-stranded RNA binding domains that bind in a sequence-independent manner to the minor groove of A-form, double stranded RNA through contacts with the $2^{\prime}-\mathrm{OH}$, and charged phosphodiester backbone with no direct contacts to any of the nucleobases [71]. Consequently, modifications to the siRNA must maintain the A-form structure, minor groove contacts, and charged phosphodiester backbone.

Second, Ago2 binds the $5^{\prime}$ terminal phosphate of the guide strand through strong mid-domain binding with multiple interactions along the phosphate backbone and $2^{\prime}-\mathrm{OH}$ in the central groove and PAZ-domain binding to the terminal $3^{\prime}$ $\mathrm{OH}[6,66,67]$. Third, structural limitations of Ago2 require at least 19 nucleotides for proper binding to both the mid and PAZ domain, with shorter oligonucleotides resulting in significantly reduced RNAi activity [72]. As a result, chemical modifications to siRNAs must maintain or closely mimic the properties of a double stranded A-form RNA with a charged phosphodiester backbone and a guide strand of at least 19 nucleotides to maintain efficient RNAi activity.

Fortunately, the siRNA backbone is amenable to some types of modifications to improve its stability and reduce activation of the innate immune system. First, incorporation of $2^{\prime}-\mathrm{F}$ and $2^{\prime}$-OMe modifications greatly reduces the ability of RNAses to degrade the siRNA. 2'-F and 2'-OMe closely mimic the biophysical properties of the 2'-OH group and are highly tolerated by TRBP and Ago [1]. In fact, most, if not all, siRNAs in clinical trials today utilize fully $2^{\prime}$ modified siRNAs $[6,73]$. However, care must be taken to prevent placement of a 2'-OMe modification on the passenger strand opposite the cleavage position, which interferes with passenger strand cleavage and loading into Ago2 [74]. 2' modifications also dramatically reduce the ability of TLR-3/7/8 to recognize the siRNA as a foreign nucleic acid invader [5,6,75].

The shift from siRNAs with limited 2'-modifications to fully $2^{\prime}$ modified siRNAs has resulted in a significant lowering of the dose curve. Second, similar to increasing the stability of ASOs, placement of single or several phosphorothioates on the extreme $5^{\prime}$ and $3^{\prime}$ ends of each strand greatly improves stability, potency, and durability of RNAi response in vivo. Terminal phosphorothioates are also well tolerated by TRBP as these locations lie outside of its minor groove binding area [71]. Third, although siRNAs differ between various groups, three general RNAi triggers have emerged: (1) the classic 21/21 nucleotide passenger/guide template with 19 base pairs and two $3^{\prime}$ nucleotide overhangs, (2) 21/23 nucleotides with a blunt end on the $5^{\prime}$ guide strand end and a 
two nucleotide $3^{\prime}$ overhang on the opposite end, and (3) dicer substrates that are longer with 26/28 nucleotides with a short single-stranded nucleotide loop near the equivalent of the $5^{\prime}$ end of the guide strand [3].

While all of siRNA modifications and structural variations have resulted in improved RNAi responses, perhaps the most significant contribution has been the fully $2^{\prime}$ modification pattern, which has primarily been driven by the chemists at Alnylam Pharmaceuticals. Initially they introduced their standard template chemistry (STC), which replaced all of the $2^{\prime}-\mathrm{OHs}$ with an alternating $2^{\prime}-\mathrm{F}$ and $2^{\prime}$-OMe pattern [76]. This was followed up with enhanced stability chemistry (ESC) [77] that improved upon STC by increasing the overall 2'-OMe content, while decreasing the $2^{\prime}$-F content $\left(2^{\prime}\right.$-OMe are more stable than $2^{\prime}$-F), as well as adding on two terminal phosphorothioates at each $5^{\prime}$ end of the passenger (STC only contained phosphorothioates on the $3^{\prime}$ end of the guide strand) [78,79].

Direct comparisons between STC and ESC using several siRNA sequences and targets showed a 5- to 10-fold higher potency for ESC siRNAs versus STC siRNAs in vivo $[7,80]$. This work was followed by yet more refinement to generate advanced ESC (or ESC+) in which the 2'-F total content is reduced to a mere 9-10 positions out of 44 with the rest being $2^{\prime}$-OMe [81]. Importantly, 2'-F must remain at key positions, including position 2 on the guide strand and a short patch on the passenger strand at the cleavage site. Together, these modifications reduced the EC50 by an additional $\sim 8$-fold and perhaps, even more importantly, resulted in a much longer duration of RNAi response [82]. Other modifications were also investigated, including incorporation of DNA, LNA, 2'-F-5'methyl modifications, $5^{\prime}$-vinylphosphonate $\left(5^{\prime}\right.$-VP) $[83,84]$, AU pairing at the $5^{\prime}$ end of the guide strand, and glycol nucleic acid modifications within the seed region. One modification in particular 5'-VP has also been shown to increase potency of modified siRNA 3- to 10-fold in vivo [83,84].

\section{Combining the Pieces of the Puzzle to Develop GaINAc-siRNA Conjugates}

While chemical modifications have greatly stabilized siRNA against RNases and innate immune responses, these modifications are still insufficient to deliver these large $(14 \mathrm{kDa})$ charged (40 phosphates) macromolecules across the lipid bilayer and into cytoplasm. Building on 50 years of ASGPR and $30+$ years of galactose delivery studies, Monoharan's group at Alnylam $[7,85]$ eloquently put together all the pieces of the puzzle and conjugated tris-GalNAc to siRNAs to show that the conjugate had significant RNAi activity in liver hepatocytes in vivo [1-3]. Unlike complicated LNP formulations, GalNAcsiRNA conjugates are a simpler, smaller, and compositionally defined approach for hepatic delivery. A complete GalNAcsiRNA can be synthesized on a solid-state oligonucleotide synthesizer [86] and chemically defined by mass spectrometry.

Early work showed that GalNAc valency and positioning played an important role in ASGPR targeting. In 1995, a neoglycopeptide YEE(ah-GalNAc)3 was used as targeting ligand to deliver a short, neutral methylphosphonate 8-mer tester oligonucleotide [57]. Linker length and sugar arrangement continued to be optimized, when in 1999, Biessen's laboratory refined a tris-Galactoside structure that was used to deliver lipids and ASOs $[60,87]$. Tris-GalNAc structure activity relationship looking at linker length and configurations was ex- tensively interrogated using ASO and siRNA conjugates in the early 2000s $[88,89]$. It was later shown that sequential conjugation of GalNAc sugars on nucleosidic linkages had similar potency to tri-antennary GalNAc conjugates, allowing for more flexible GalNAc delivery platforms [86,90,91]. The GalNAc approach was subsequently shown to enhance hepatocyte delivery of ASOs by $\sim 10$-fold versus free ASOs in preclinical models, resulting in a dramatic dose reduction [58].

The high number, rapid turnover, and recycling of ASGPR receptors are thought to contribute to GalNAc delivery of siRNAs. GalNAc-siRNA conjugates bind ASGPR and are rapidly internalized into clathrin-coated endosomes (Fig. 1). As the endosomal $\mathrm{pH}$ drops, the GalNAc-siRNA is released from binding ASGPR. ASGPR is recycled back to the cell surface, while the GalNAc-siRNA remains in the lumen of the endosome. GalNAc is cleaved from the siRNA by endosomal glycosidases by $1 \mathrm{~h}$, and the linker arms are degraded by $4 \mathrm{~h}$ [58].

The vast majority of free siRNA remains trapped in the endosome, while a very small amount $(<1 \%)$ is able to traverse the endosomal lipid bilayer membrane through an unknown mechanism to enter the cytoplasm and induce an RNAi response (Fig. 1). In addition to the mystery of how the siRNA actually escapes the endosome to enter the cytoplasm, which is the ratelimiting step, it is unknown how increasing the 2 -OMe content increases the duration of the RNAi response. These mechanistic details aside, together, GalNAc-siRNA and GalNAc-ASO conjugate delivery studies performed by many groups have shown the exquisite superiority overall for this delivery approach.

\section{Success of GalNAc-siRNA in Clinical Trials}

The following three biotech companies are currently performing clinical trials using GalNAc-siRNA conjugates: Alnylam Pharmaceuticals, Arrowhead Pharmaceuticals, and Dicerna Pharmaceuticals. While there were several significant bumps early along the road, overall data from the next generation RNAi triggers look nothing short of spectacular.

Alnylam currently has six GalNAc-siRNA conjugates in clinical trials, including three phase III trials for wide variety of liver hepatocyte diseases. Alnylam performed the first GalNAc-siRNA conjugate clinical trials with Revusiran (ALN-TTRsc), which targets the transthyretin (TTR) gene to treat TTR-mediated amyloidosis (D2, D1-64, D1-65). Phase I clinical trials administered subcutaneous single ascending dose and multiple ascending dose (MAD) in a 2:1 drug to placebo randomized patient population. However, 9 out of 19 MAD patients showed a dose independent elevation of liver aspartate aminotransferase (AST) and/or alanine aminotransferase (ALT) [92]. Levels of AST and ALT returned to normal with continued dosing, and no antidrug antibodies were observed in the MAD treatment group.

A phase II and open label extension (OLE) reported $\sim 90 \%$ reduction of TTR serum levels after multiple dosing with a sustained knockdown of TTR beyond 90 days in hereditary ATTR patients with cardiomyopathy [93,94]. Following a 12-month treatment, five of nine patients had met the primary endpoint goal of a stable 6 min walk distance. However, phase II OLE data revealed that $20 \%$ of Revusiran treated patients experienced peripheral neuropathy, prompting Alnylam to request an ad hoc investigation of their ongoing phase III ENDEAVOUR study by a Data Monitoring Committee (DMC) [95]. The DMC found an imbalance in 
mortality of 16 deaths in the Revusiran arm compared to 2 deaths in the placebo arm (2:1 drug to placebo patient distribution), leading Alnylam to discontinue all ongoing Revusiran dosing on October 5, 2016. Ultimately, the DMC found no conclusive evidence for drug related neuropathy, but could not exclude the possibility of a drug related effect on mortality in the ENDEAVOUR study [95].

Despite the discontinuation of Revusiran, the ionizable LNP based sister product, Patisiran, targeting TTR has completed a phase III APOLLO study, and continued long-term treatment in a global OLE study demonstrates continued safety and efficacy of liver targeted TTR knockdown [96]. Patisiran will, in all likelihood, become the first approved siRNA therapeutic.

Revusiran utilized the less stable STC backbone, which required high and frequent dosing, resulting in extensive patient exposure to the drug [95]. However, all subsequent GalNAc-siRNA conjugate clinical trials use the much more stable and more active ESC platform (see discussion above). The follow on for TTR is ALN-TTRsc02 which has completed a phase I clinical trial. A randomized ascending fixed dose (5-300 mg) in 80 healthy volunteers reported a maximum mean TTR knockdown of $97 \%$ maintained over 320 days. No Severe Adverse Events (SAEs) or study discontinuations due to Adverse Events (AE) were reported; AEs remain high in both arms (77\% for ALN-TTRsc02 and $50 \%$ for placebo), likely due to the nature of the TTR disease [97].

Due to the dramatically increased stability and duration of RNAi responses by ESC siRNAs, ALN-TTRsc02 knockdown of TTR supports a low dose of $25 \mathrm{mg}$ /quarter to achieve comparable knockdown by Patisiran (LNP delivery). The projected dose for ALN-TTRsc02 represents an annualized dose of $100 \mathrm{mg}$ compared to $28 \mathrm{~g}$ for Revusiran. Alnylam has reported the incidence of $\mathrm{AE}$ and $\mathrm{SAE}$ across their platform as of 2016 and found low incidence of AE (15.2\%) consisting of mild transient injection site reactions, as well as a low incidence $(2.2 \%)$ of SAE consisting of mild, asymptomatic, and reversible liver function test (LFT) increases $>3$-fold above upper limit of normal [95]. Alnylam continues to see this SAE across several of its RNAi drugs. Further advances in stability chemistry that led to the ESC+ platform are expected to reduce these SAEs. Alnylam has announced that its first ESC+ platform candidate, ALN-AAT02, will enter clinical trials late 2018, with an anticipated further improvement in dosing and safety profile expected over current ESC candidates [98].

Givosiran (ALN-AS1) $[99,100]$ is a GalNAc-siRNA conjugate that targets the ALAS1 gene to treat acute hepatic porphyria [101,102]. Givosiran has completed a phase I clinical trial and is currently under both a phase I/II OLE and a phase III ENVISION trial. Phase I clinical trial data revealed no SAEs attributed to Givosiran with subcutaneous doses as high as $5 \mathrm{mg} / \mathrm{kg}$ monthly. Patients dosed monthly compared to quarterly with $2.5 \mathrm{mg} / \mathrm{kg}$ saw an enhanced lowering of target biomarkers, aminolevulinic acid (ALA) and porphobilinogen (PBG). Increasing the monthly dose to $5 \mathrm{mg} / \mathrm{kg}$ did not show increased reduction of ALA and PBG levels. Monthly dosing of $2.5 \mathrm{mg} / \mathrm{kg}$ led to an $83 \%$ reduction in annualized attack rate (AAR) and $88 \%$ reduction in hemin use relative to placebo.

Preliminary results from an ongoing phase I/II OLE trial using $2.5 \mathrm{mg} / \mathrm{kg}$ monthly injection reveal an enhanced clinical activity in patients treated with Givosiran up to 22 months with increased reductions in AAR and hemin use of 93\% and 94\%, respectively. Patients who have crossed over from the placebo arm have shown mean reductions in AAR and hemin use greater than $90 \%$ compared to run-in and placebo treatment periods. After a mean treatment period of 8.5 months, 7 of 16 patients in the OLE study have achieved an AAR of zero compared to an AAR of 15.1 for the same patients before treatment. A phase III clinical trial ENVISION is enrolling and currently ongoing. Givosiran has been granted Breakthrough Therapy designation by the U.S. Food and Drug Administration (FDA), PRIME designation by the European Medicines Agency, and orphan disease designations in both the United States and EU [101,102].

Fitusiran (ALN-AT3sc) targets antithrombin 3 (AT3) for the treatment of Hemophilia A and B [103,104]. Completion of a phase I study showed no drug related SAEs and a reduction in AT of 70\%-89\% following monthly dosing of $0.225-1.8 \mathrm{mg} / \mathrm{kg}$, resulting in peak thrombin levels within the lower range of healthy participants [104]. A phase II OLE study dosing patients for a maximum of 20 months and a median of 11 months showed a reduction in AT of $80 \%$ with only two drug related SAEs observed in patients with preexisting conditions [105].

In all patients who received Fitusiran, thrombin levels remained at the lower end of normal for the duration of observation. Annualized bleeding rate (ABR) was zero in the Fitusiran + inhibitor (factor VII or bypassing agents) group compared to an ABR of 20 in the Fitusiran only group and an $\mathrm{ABR}$ of 38 in the inhibitor only group [105]. Impressively, $48 \%$ of Fitusiran + inhibitor patients remained bleed free for the duration of observation, and $67 \%$ of patients experienced zero spontaneous bleeds. In a separate, smaller phase study, patients receiving Fitusiran required reduced dosing of FVII or bypassing agents for perioperative management following dental work [103]. The ATLAS phase III study is currently enrolling to further study Fitusiran's safety and efficacy.

Inclisiran (ALN-PCSsc) [106,107] is a first-in-class PCSK9 synthesis inhibitor for treating hypercholesterolemia. Inclisiran completed a phase II ORION-1 study, which was the largest randomized, placebo controlled study for an investigational RNAi therapeutic [95]. Patients were dosed with $300 \mathrm{mg}$ Inclisiran subcutaneously at day 1 and 90, which resulted in a time averaged reduction in LDL cholesterol (LDL-C) of $51 \%$ over the following 6-month period (day 90-270) [108,109]. No drug related differences in LFT were observed between the Inclisiran and placebo arms [95].

Following these positive results, Alnylam is moving forward with a phase III trial using a $300 \mathrm{mg}$ maintenance dose every 6 months following initial dosing [108,109]. In contrast, hypercholesterolemia patients currently taking statins are required to dose every day versus Inclisiran's anticipated twice yearly dosing. Alnylam's clinical pipeline also has two early clinical trial GalNAc-siRNA conjugates. Lumasiran (ALN-GO1) targets glycolate oxidase (GO) for treatment of primary hyperoxaluria (PH) type 1 [110-112]. Cemdisiran (ALN-CC5) targets complement component C5 (CC5) for the treatment of complement-mediated disease [113].

Arrowhead Pharmaceuticals uses two types of GalNAc conjugates. The first generation was a dynamic polyconjugate (DPC) that utilized an endosomolytic peptide (butyl and amino vinyl ether, PBAVE, or melittin) masked with GalNAc through a $\mathrm{pH}$ sensitive carboxy dimethyl maleic anhydride linkage $[114,115]$. The siRNA is conjugated to cholesterol and is co-injected with the DPC as combination therapy. The 
cholesterol-siRNA forms a large aggregate (low-density lipoprotein) in blood, which is transported to and taken up by the liver, whereas the GalNAc-DPC is taken up specifically by ASGPR in the liver. When both are present in the same endosome, the GalNAc-DPC facilitates endosomal escape of the cholesterol-siRNA conjugates [114,115].

Arrowhead's lead compound into the clinics was ARC-520 along with a related ARC-521, which contained two siRNAs targeting different regions in the $\mathrm{X}$ gene in Hepatitis- $\mathrm{B}$ infection (HBV) [116,117]. ARC-520/521 has undergone multiple phase I and phase II trials alone and in combination with the antiviral entecavir [118]. However, in late 2016, the FDA halted five ongoing clinical trials involving ARC-520 due to a NHP death in one of Arrowhead's preclinical studies, likely due to toxicity from the DPC [119]. Despite the termination of the clinical trials, results from the clinical trials showed a rapid, six log suppression ( $>99.9 \%$ ) of HBV DNA in all Hepatitis $\mathrm{B}$ e-antigen positive $(\mathrm{HBe} \mathrm{Ag})$ treatment naive patients.

Treatment of $\mathrm{HBeAg}$-negative naive patients reduced HBV DNA below the limit of detection. Single dose treatment of ARC-520 also inhibited covalently closed circular (cccDNA)-derived mRNA expression and reduced viral protein production by $99 \%$. In addition, some patients developed and expressed antibodies against hepatitis B surface antigen [117]. Despite the regulatory shutdown of the ARC520/521 clinical trials, Arrowhead showed that HBV is susceptible to RNAi therapeutics and gleaned a large volume of clinical data on just how to best tackle HBV.

Arrowhead's next generation delivery platform, called Targeted RNAi Molecule (TRiM), removes the problematic active endosomal escape agent (PBAVE, melittin) in favor of direct conjugation of GalNAc targeting domains [120]. While the exact nature of the TRiM siRNAs has not been made public, Arrowhead reports that numerous tailor design chemistries have been incorporated to generate highly robust RNAi triggers. Building off of their prior RNAi clinical experience, ARO-HBV targets both the $\mathrm{X}$ gene and the $\mathrm{S}$ gene. Importantly, the $\mathrm{X}$ gene targeting sequence is present on all integrated forms of HBV, whereas the ARC-520 siRNAs did not.

Using multiple sequence targets present on both cccDNA and integrated $\mathrm{HBV}, \mathrm{ARO}-\mathrm{HBV}$ reduces the opportunity for HBV to develop resistance to the RNAi therapeutics and allows the drug to tackle both forms of the virus [120], which is critical for achieving a cure. Arrowhead has begun recruiting patients for phase I/II clinical trials for ARO-HBV. Arrowhead also has begun recruitment for a phase I clinical trial involving ARO-AAT to treat alpha-1 antitrypsin (AAT) related liver disease by knocking down the mutant AAT gene [121]. In addition, Arrowhead has several preclinical GalNAc TRiM programs moving toward the clinics in 2018/2019.

Dicerna Pharmaceuticals has developed a proprietary GalNAc delivery platform that differs from both Alnylam and Arrowhead. Dicerna's GalXC technology utilizes an altered siRNA structure and tetra-antennary GalNAc, rather than the more widely used tri-antennary pattern [122]. Monomeric GalNAcs are covalently linked to four nucleotides on the single stranded loop of their dicer substrate siRNAs [122]. The Guide strand is annealed to this altered passenger strand forming a mature GalNAc-dicer substrate siRNA conjugate containing a nick at the $5^{\prime}$ end of the guide strand separating it from the looped passenger strand sequence. This approach provides a simple "on column" oligo manufacturing with proper orientation of the four GalNAc ligands. Using this GalXC platform, Dicerna has developed several preclinical candidates with one, DCR-PHXC, advancing to phase I clinical trials for the treatment of PH. Dicerna also has multiple GalNAc-siRNA conjugates in preclinical development.

\section{Conclusions}

The discovery of ASGPR by Ashwell and Morell over 50 years ago provided a treasure trove for the treatment of human disease. GalNAc represents a powerful, long-lasting, and low toxicity approach for the delivery of a diverse array of cargos, including RNAi triggers and ASOs. Current clinical trials show significant progress toward safe and efficacious treatments with patient dosing far less frequent than conventional small molecule therapies, which for hypercholesteremia is twice yearly versus daily. It is now fair to state that siRNA delivery to liver hepatocytes has been solved. However, this would not be so if it were not for 50 years of nucleic acid chemistry that has been applied to siRNAs to increase stability, potency, duration of response, and avoidance of the innate immune system.

Unlike new small molecule therapeutics where one is concerned about Pharmacokinetics (PK), Pharmacodynamics (PD), toxicology, and validating the right target for the disease, the only relevant question for new GalNAc-siRNA conjugates is have you chosen the right target gene, because knocking it down is a given as is a consistent PK. While the lessons learned in siRNA design chemistries will be applicable to extrahepatic delivery approaches, unfortunately, ASGPR appears to be a one of a kind miracle for delivery. GalNAc-siRNA conjugates escape the endosome in an unknown and rate-limiting mechanism that may be prohibitively infrequent in systems lacking the high receptor number and rapid turnover that is seen with ASGPR. Consequently, just like plastics in the 1960s [123], solving the endosomal escape problem-that's the future!

\section{Acknowledgment}

The authors thank EY027507-01 for funding.

\section{Author Disclosure Statement}

S.F.D. is a founder of Solstice Biologics. A.D.S. declares no competing financial interests exist.

\section{References}

1. Dowdy SF. (2017). Overcoming cellular barriers for RNA therapeutics. Nat Biotechnol 35:222-229.

2. Juliano RL. (2016). The delivery of therapeutic oligonucleotides. Nucleic Acids Res 44:6518-6548.

3. Khvorova A and JK Watts. (2017). The chemical evolution of oligonucleotide therapies of clinical utility. Nat Biotechnol 35:238-248.

4. Zimmermann TS, AC Lee and A Akinc. (2006). RNAimediated gene silencing in non-human primates. Nature 44:111-114.

5. Gantier MP and BR Williams. (2007). The response of mammalian cells to double-stranded RNA. Cytokine Growth Factor Rev 18:363-371.

6. Rerrig GR and MA Behlke. (2012). Progress toward in vivo use of siRNAs-II. Mol Ther 20:483-512. 
7. Morell AG, G Gregoriadis, IH Scheinberg, J Hickman and G Ashwell. (1971). The role of sialic acid in determining the survival of glycoproteins in the circulation. J Biol Chem 246:1461-1467.

8. Nair JK, JLWilloughby, A Chan, K Charisse, MR Alam, Q Wang, M Hoekstra, P Kandasamy, AV Kel'in, et al. (2014). Multivalent $\mathrm{N}$-acetylgalactosamine-conjugated siRNA localizes in hepatocytes and elicits robust RNAi-mediated gene silencing. J Am Chem Soc 136:16958-16961.

9. Grewal PK. (2010). The Ashwell-Morell receptor. Methods Enzymol 479:223-241.

10. Stockert RJ, AG Morell and IH Scheinberg. (1974). Mammalian hepatic lectin. Science 186:365-366.

11. Anonymous. (2008). Gilbert Ashwell: sweet on science. Nat Med 14:608.

12. Morell AG, CJ van den Hamer, IH Scheinberg and G Ashwell. (1966). Physical and chemical studies on ceruloplasmin. IV. Preparation of radioactive, sialic acid-free ceruloplasmin labeled with tritium on terminal D-galactose residues. J Biol Chem 241:3745-3749.

13. Morell AG, RA Irvine, I Sternlieb, H Scheinberg and G Ashwell. (1968). Physical and chemical studies on ceruloplasmin. V. Metabolic studies on sialic acid-free ceruloplasmin in vivo. J Biol Chem 243:155-159.

14. Pricer WE, Jr., and G Ashwell. (1971). The binding of desialylated glycoproteins by plasma membranes of rat liver. J Biol Chem 246:4825-4833.

15. Morell AG and IH Scheinberg. (1972). Solubilization of hepatic binding sites for asialo-glycoproteins. Biochem Biophys Res Commun 48:808-815.

16. Sarkar M, J Liao, EA Kabat, T Tanabe and G Ashwell. (1979). The binding site of rabbit hepatic lectin. J Biol Chem 254:3170-3174.

17. Novogrodsky A and G Ashwell. (1977). Lyphocyte mitogenesis induced by a mammalian liver protein that specifically binds desialylated glycoproteins. Cell Biol 74:676-678.

18. Hickman J, G Ashwell, AG Morell, CJ van den Hamer and IH Scheinberg. (1970). Physical and chemical studies on ceruloplasmin. 8. Preparation of $\mathrm{N}$-acetylneuraminic acid1-14C-labeled ceruloplasmin. J Biol Chem 245:759-766.

19. Van den Hamer CJA, AG Morell, IH Scheinberg, J Hickman and G Ashwell. (1970). Physical and chemical studies on ceruloplasmin. IX. The role of galactosyl residues in the clearance of ceruloplasmin from the circulation. J Biol Chem 245:4397-4402.

20. van Lenten L and G Ashwell. (1972). The binding of desialylated glycoproteins by plasma membranes of rat liver. Development of a quantitative inhibition assay. J Biol Chem 247:4633-4640.

21. Kawaguchi K, M Kuhlenschmidt, S Roseman and YC Lee. (1980). Synthesis of some cluster galactosides and their effect on the hepatic galactose binding system. Arch Biochem Biophys 205:388-395.

22. Steer CJ and G Ashwell. (1980). Studies on a mammalian hepatic binding protein specific for asialoglycoproteins. Evidence for receptor recycling in isolated rat hepatocytes. J Biol Chem 255:3008-3013.

23. Lee YC, RR Townsend, MR Hardy, J lönngren, J Arnarp, M Haralsson and H Lönn. (1982). Binding of synthetic oligosaccharides to hepatic Gal/GalNAc lectin. J Biol Chem 258:199-202.

24. Baenziger AU and D Fiete. (1980). Galactose and $N$ acetylgalactosamine-specific endocytosis of glycopeptides by isolated rate hepatocytes. Cell 22:611-620.
25. Lee YC and RT Lee. (2008). Interactions of oligosaccharides and glycoproteins with hepatic carbohydrate receptors. In: Carbohydrates in Chemistry and Biology. Vol. 4. Ernst B, GW Hart, P Sináy, eds. Wiley-VCH Verlag GmBh, Weinheim, Germany, pp 549-561.

26. Meier M, MD Bider, VN Malashkevich, $M$ Spiess and $P$ Burkhard. (2000). Crystal structure of the carbohydrate recognition domain of the $\mathrm{H} 1$ subunit of the asialoglycoprotein receptor. J Mol Biol 300:857-865.

27. Mammen M, S-K Choi and GM Whitesides. (1998). Polyvalent interactions in biological systems: implications for design and use of multivalent ligands and inhibitors. Agnew Chem Int Ed 37:2754-2794.

28. D'Sousa AA and PV Devarajan. (2015). Asialoglycoprotein receptor mediated hepatocyte targeting-strategies and applications. J Control Release 203:126-139.

29. Schwartz AL, A Marshak-Rothstein, D Rup and HF Lodish. (1981). Identification and quantification of the rate hepatocyte asialoglycoprotein receptor. Proc Natl Acad Sci U S A 78:3348-3352.

30. Schwartz AL, D Rup and HF Lodish. (1980). Difficulties in the quantification of asialoglycoprotein receptors on the rat hepatocyte. J Biol Chem 255:9033-9036.

31. Pricer WE and G Ashwell. (1976). Subcellular distribution of a mammalian hepatic binding protein specific for asialoglycoproteins. J Biol Chem 251:7539-7544.

32. Kolb-Bachofen V. (1981). Hepatic receptor for asialoglycoproteins. Ultrastructural demonstration of ligandinduced microaggregation of receptors. Biochim Biophys Acta 645:293-299.

33. Weigel PH. (1980). Rat hepatocytes bind to synthetic galactoside surfaces via a patch of asialoglycoprotein receptors. J Cell Biol 87:855-861.

34. Schwartz AL, SE Fridovich and HF Lodish. (1982). Kinetics of internalization and recycling of the asialoglycoprotein receptor in a hepatoma cell line. J Biol Chem 257:4230-4237.

35. Stockert RJ, HB Haimes, AG Morell, PM Novikoff, AB Novikoff, N Quintana and I Sternlieb. (1980). Endocytosis of asialoglycoprotein-enzyme conjugates by hepatocytes. Lab Invest 43:556-563.

36. Tanabe T, WE Pricer, Jr., and G Ashwell. (1979). Subcellular membrane topology and turnover of rat hepatic binding protein specific for asialoglycoproteins. J Biol Chem 254:1038-1043.

37. Gregoriadis G, AG Morell, I Sternlieb and IH Scheinberg. (1970). Catabolism of desialylated ceruloplasmin in the liver. J Biol Chem 245:5833-5837.

38. Bridges K, J Harford, G Ashwell and RD Klausner. (1982). Fate of receptor and ligand during endocytosis of asialoglycoproteins by isolated hepatocytes. Proc Natl Acad Sci U S A 79:350-354.

39. Wall DA, G Wilson and AL Hubbard. (1980). The galactose-specific recognition system of mammalian liver: the route of ligand internalization in rat hepatocytes. Cell 21:79-93.

40. Geuze HJ, JW Slot, GJ Strous, HF Lodish and AL Schwartz. (1983). Intracellular site of asialoglycoprotein receptor-ligand uncoupling: double-label immunoelectron microscopy and during receptor-mediated endocytosis. Cell 32:277-287.

41. Rogers JC and S Kornfield. (1971). Hepatic uptake of proteins coupled to fetuin glycopeptide. Biochem Biophys Res Commun 45:622-629. 
42. Wilson G. (1978). Effect of reductive lactosamination on the hepatic uptake of bovine pancreatic ribonuclease A dimer. J Biol Chem 253:2070-2072.

43. Fiume L, A Mattioli, PG Balboni, M Tognon, G BarbantiBrodano, J de Vries and T Wieland. (1979). Enhanced inhibition of virus DNA synthesis in hepatocytes by trifluorothymidine coupled to asialofetuin. FEBS Lett 103: 47-51.

44. Fiume L, A Mattioli, C Busi, PG Balboni, G BarbantiBrodano, J de Vries, R Altmann and T Wieland. (1980). Selective inhibition of ectromelia virus DNA synthesis in hepatocytes by adenine-9- $\beta$-D-arabinofuranoside (ARAA) and adenine-9- $\beta$-arabinofuranoside $5^{\prime}$-monophosphate (ARA-AMP) conjugated to asialofetuin. FEBS Lett 116: 185-188.

45. Attie AD, RC Pittman and D Steinberg. (1980). Metabolism of native and lactosylated human low density lipoprotein: evidence for two pathways for catabolism of exogenous proteins in rat hepatocytes. Proc Natl Acad Sci U S A 77:5923-5927.

46. Simpson DL, DB Cawley and HR Herschman. (1982). Killing of cultured hepatocytes by conjugates of asialofetuin and EGF linked to the A chains of ricin and diphtheria toxin. Cell 29:469-473.

47. Rensen PCN, SH van Leeuwen, LA Sliedregt, TJC van Berkel and EA Biessen. (2004). Design and synthesis of novel $\mathrm{N}$-acetylgalactosamine-terminated glycolipids for targeting of lipoproteins to the hepatic asialoglycoprotein receptor. J Med Chem 47:5798-5808.

48. Seymour LW, DR Ferry, D Anderson, S Hesslewood, PJ Julyan, R Poyner, J Doran, AM Young, S Burtles, DJ Kerr and Cancer Research Campaign Phase I/II Clinical Trials Committee. (2002). Hepatic drug targeting: phase I evaluation of polymer bound doxorubicin. J Clin Oncol 20: 1668-1676.

49. Rohlff C, SA Watson, TM Morris, L Skelton, AL Jackman and MJ Page. (1999). A novel, orally administered nucleoside analogue, OGT 719, inhibits liver invasive growth of human colorectal tumor, C170HM2. Cancer Res 59:1268-1272.

50. Fiume L, C Busi and A Mattioli. (1983). Targeting antiviral drugs by coupling with protein carriers. FEBS Lett 153:6-10.

51. Wu GY and CH Wu. (1987). Receptor-mediated in vitro gene transformation by soluble DNA carrier system. J Biol Chem 262:4429-4432.

52. Wu GY and CH Wu. (1988). Receptor-mediated gene delivery and expression in vivo. J Biol Chem 263:14621-14624.

53. Wu GY and $\mathrm{CH}$ Wu. (1988). Evidence for targeted gene delivery to Hep G2 hepatoma cells in vitro. Biochimie 27: 887-892.

54. Plank C, K Zatloukal, M Cotten, K Mechtler and E Wagner. (1992). Gene transfer into hepatocytes using asialoglycoprotein receptor mediated endocytosis of DNA complexed with artificial terta-antennary galactose ligand. Bioconjug Chem 3:533-539.

55. Merwin JR, GS Noell, WL Thomas, HC Chiou, M DeRome, TD McKee, GL Spitalny and MA Findeis. (1994). Targeted delivery of DNA using YEE(GalNAcAH)3, a synthetic glycopeptide ligand for the asialoglycoprotein receptor. Bioconjug Chem 5:612-620.

56. Wu GY and CH Wu. (1992). Specific inhibition of hepetitis B viral gene expression in vitro by targeted antisense oligonucleotides. J Biol Chem 267:12436-12439.

57. Hangeland JJ, JT Levis, YC Lee and PO Ts'o. (1995). Cell-type specific and ligand specific enhancement of cellular uptake of oligodeoxynucleoside methylphosphonates covalently linked with neoglycopeptide, YEE(ah-GalNAC)3. Bioconjug Chem 6:695-701.

58. Prakash TP, MJ Graham, J Yu, R Carty, A Low, A Chappell, K Schmidt, C Zhao, M Aghajan, et al. (2014). Targeted delivery of antisense oligonucleotides to hepatocytes using triantennary $\mathrm{N}$-acetylgalactosamine improved potency 10 fold in mice. Nucleic Acid Res 42:8696-8807.

59. Yu RZ, MJ Graham, N Post, S Riney, T Zanardi, S Hall, J Burkey, CS Shemesh, TP Prakash, et al. (2016). Disposition and pharmacology of GalNAc3-conjugated ASO targeting human liopoprotein (a) in mice. Mol Ther Nucleic Acids 5:e317.

60. Biessen EA, H Vietsch, ET Rump, K Fluiter, J Kuiper, MK Bijsterbosch and TJ van Berkel. (1999). Targeted delivery of oligodeoxynucleotides to parenchymal liver cells in vivo. J Biol Chem 340:783-792.

61. Wu CH, JM Wilson and GY Wu. (1989). Targeting genes: delivery and persisten expression of a forign gene driven by mammalian regulatory elements in vivo. $\mathrm{J}$ Biol Chem 264:16985-16987.

62. Orrantia E and PL Chang. (1990). Intracellular distribution of DNA internalization through calcium phosphate precipitation. Exp Cell Res 190:170-174.

63. Fire A, S Xu, MK Montgomery, SA Kostas, SE Driver and CC Mello. (1998). Potent and specific genetic interference by double-stranded RNA in Caenorhabditis elegans. Nature 391:806-811.

64. Elbashir SM, J Harborth, W Lendeckel, A Yalcin, K Weber and T Tuschl. (2001). Duplexes of 21-nucleotide RNAs mediate RNA interference in cultured mammalian cells. Nature 411:494-498.

65. Ohrt T, J Muetze, P Svoboda and P Schwille. (2012). Intracellular localization and routing of miRNA and RNAi pathway components. Curr Top Med Chem 12:79-88.

66. Schirle NT and IJ MacRae. (2012). The crystal structure of human Argonaute1. Science 336:1037-1040.

67. Schirle NT, J Sheu-Gruttadauria and IJ MacRae. (2014). Structural basis for microRNA targeting. Science 346: 608-613.

68. Salomon WE, SM Jolly, MJ Moore, PD Zamore and V Serebrov. (2015). Single-molecule imaging reveals that Argonaute reshapes the binding properties of its nucleic acid guides. Cell 162:84-95.

69. Iversen F, C Yang, F Dagnæs-Hansen, DH Schaffert, J Kjems and S Gao. (2013). Optimized siRNA-PEG conjugates for extended blood circulation and reduced urine excretion in mice. Theranostics 3:201-209.

70. Juliano RL, X Ming, K Carver and B Laing. (2014). Cellular uptake and intracellular trafficking of oligonucleotides: implications for oligonucleotide pharmacology. Nucleic Acid Ther 24:101-113.

71. Rytler JM and SC Schultz. (1998). Molecular basis of double-stranded RNA-protein interactions: structure of a dsRNA-binding domain complexed with dsRNA. EMBO J 17:7505-7513.

72. Hagopian JC, AS Hamil, A van den Berg, BR Meade, A Eguchi, C Palm-Apergiand and SF Dowdy. (2017). Induction of RNAi responses by short left-handed hairpin RNAi triggers. Nucleic Acid Ther 27:260-271.

73. Fitzgerald K, S White, A Borodovsky, BR Bettencourt, A Strahs, V Clausen, P Wijngaard, JD Horton, J Taubel, et al. (2017). A highly durable RNAi therapeutic inhibitor of PCSK9. N Engl J Med 376:41-51. 
74. Matranga C, Y Tomari, C Shin, DP Bartel and PD Zamore. (2005). Passenger strand cleavage facilitates assembly of siRNA into Ago2-containing RNAi enzyme complexes. Cell 123:607-620.

75. Robbins M, A Judge and I MacLachlan. (2009). SiRNA and innate immunity. Oligonucleotides 19:89-102.

76. Huang Y. (2017). Preclinical and clinical advances of GalNAcdecorated nucleic acid therapeutics. Mol Ther 6:116-132.

77. Huang YY and ZC Liang. (2015). Asialoglycoprotein receptor and its application in liver-targeted drug delivery. Prog Biochem Biophys 42:501-510.

78. Rajeev KG, T Zimmermann, M Manoharan, M Maier, S Kuchimanchi and K Charisse. (2013). Modified RNAi agents. U.S. Patent WO2013074974.

79. Maier M, D Foster, S Milstein, S Kuchimanchi, V Jadhav, K Rajeev, M Manoharan and R Parmar. (2016). Modified double-stranded RNA agents. U.S. Patent WO2016028649.

80. Alnylam. (2014). Advances in delivery of RNAi therapeutics with enhanced stabilization chemistry (ESC)-GalNAc-siRNA conjugates, http://alnylam.com/web/assets/Roundtable_ESCGalNAc-Conjugates_072214.pdf Accessed on April 23, 2018.

81. Schlegel MK, DJ Foster, AV Kel'in, I Zlatev, A Bisbe, M Jayaraman, JG Lackey, KG Rajeev, K Charissé, et al. (2017). Chirality dependent potency enhancement and structural impact of glycol nucleic acid modification on siRNA. J Am Chem Soc 139:8537-8546.

82. Alnylam. (2017). Platform advances in RNAi therapeutics, http://alnylam.com/wp-content/uploads/2017/ 08/RNAi-Platform-RoundTable_FINAL20170823.pdf Accessed on April 23, 2018.

83. Parmar R, JL Willoughby, J Liu, DJ Foster, B Brigham, CS Theile, K Charisse, A Akinc, E Guidry, et al. (2016). 5'-(E)-vinylphosphonate: a stable phosphate mimic can improve the RNAi activity of siRNA-GalNAc conjugates. ChemBioChem 17:985-989.

84. Prakash TP, GA Kinberger, HM Murray, A Chappell, S Riney, MJ Graham, WF Lima, EE Swayze and PP Seth. (2016). Synergistic effect of phosphorothioate, 5 -vinylphosphonate and GalNAc modifications for enhancing activity of synthetic siRNA. Bioorg Med Chem Lett 26:2817-2820.

85. Sehgal A, S Barros, L Ivanciu, B Cooley, J Qin, T Racie, J Hettinger, M Carioto, Y Jiang, et al. (2015). An RNAi therapeutic targeting antithrombin to rebalance the coagulation system and promote hemostasis in hemophilia. Nat Med 21:492-497.

86. Rajeev KG, JK Nair, M Jayaraman, K Charisse, N Taneja, J O'Shea, JL Willoughby, K Yucius, T Nguyen, et al. (2015). Hepatocyte-specific delivery of siRNAs conjugated to novel non-nucleosidic trivalent $\mathrm{N}$-acetylgalactosamine elicits robust gene silencing in vivo. ChemBioChem 16:903-908.

87. Sliedregt LA, PC Rensen, ET Rump, PJ van Santbrink, MK Bijsterbosch, AR Valentijn, GA van der Marel, JH van Boom, TJ van Berkel and EA Biessen. (1999). Design and synthesis of novel amphiphilic dendritic galactosides for selective targeting of liposomes to the hepatic asialoglycoprotein receptor. J Med Chem 42:609-618.

88. Prakash TP, J Yu, MT Migawa, GA Kinberger, WB Wan, ME Østergaard, RL Carty, G Vasquez, A Low, et al. (2016). Comprehensive structure-activity relationship of triantennary $\mathrm{N}$-acetylgalactosamine conjugated antisense oligonucleotides for targeted delivery to hepatocytes. J Med Chem 59:2718-2733.

89. Migawa MT, TP Prakash, G Vasquez, WB Wan, J Yu, GA Kinberger, ME Østergaard, EE Swayze and PP Seth.
(2016). A convenient synthesis of $5^{\prime}$-triantennary $N$-acetylgalactosamine clusters based on nitromethanetrispropionic acid. Bioorg Med Chem Lett 26:2194-2197.

90. Matsuda S, K Keiser, JK Nair, K Charisse, RM Manoharan, P Kretschmer, CG Peng, AV Kel'in, P Kandasamy, et al. (2015). siRNA conjugates carrying sequentially assembled trivalent $\mathrm{N}$-acetylgalactosamine linked through nucleosides elicit robust gene silencing in vivo in hepatocytes. ACS Chem Biol 10:1181-1187.

91. Sebestyén MG, SC Wong, V Trubetskoy, DL Lewis and CI Wooddell. (2015). Targeted in vivo delivery of siRNA and an endosome-releasing agent to hepatocytes. Methods Mol Biol 1218:163-186.

92. Zimmermann TS, V Karsten, A Chan, J Chiesa, M Boyce, BR Bettencourt, R Hutabarat, S Nochur, A Vaishnaw and J Gollob. (2017). Clinical proof of concept for a novel hepatocytetargeting GalNAc-siRNA conjugate. Mol Ther 25:71-78.

93. Hawkins PN, Y Ando, A Dispenzeri, A Gonzalez-Duarte, D Adams and OB Suhr. (2015). Evolving landscape in the management of transthyretin amyloidosis. Ann Med 47: 625-638.

94. Butler JS, A Chan, S Costelha, S Fishman, JLS Willoughby, TD Borland, S Milstein, DJ Foster, P Gonçalves, et al. (2016). Preclinical evaluation of RNAi as a treatment for transthyretin-mediated amyloidosis. Amyloid 23:109-118.

95. Alnylam. (2017). Investigation of mortality imbalance in Revusiran phase 3 study, ENDEAVOUR, http://investors .alnylam.com/static-files/c8f6284e-841b-4f0e-846d-046aa 73824b6 Accessed on April 23, 2018.

96. Alnylam. (2018). Alnylam presents new clinical results from the Apollo Phase 3 study of Patisiran at the 16th international symposium on amyloidosis, http://investors .alnylam.com/news-releases/news-release-details/alnylampresents-new-clinical-results-apollo-phase-3-study Accessed on April 23, 2018.

97. Taubel J, T Zimmermann, V Karsten, C Martinez, A Chan, Y Wang, H Attarwala, J Gollob and J Vest. (2018). Phase 1 study of ALN-TTRsc02, a subcutaneously administered investigational RNAi therapeutic for the treatment of transthyretin-mediated amyloidosis, http://alnylam.com/ wp-content/uploads/2018/03/10.-TTR-SCO2_FINAL.pdf Accessed on April 23, 2018.

98. Alnylam. (2018). Nonclinical research published in nature communications describing optimization of GalNAcsiRNA conjugates to minimize off-target potential and maximize therapeutic index, http://alnylam.com/2018/02/ 19/nonclinical-research-published-in-nature-communicationsdescribing-optimization-of-galnac-sirna-conjugates-to-min imize-off-target-potential-and-maximize-therapeutic-index/ Accessed on April 23, 2018.

99. Yasuda M, L Gan, B Chen, S Kadirvel, C Yu, JD Phillips, MI New, A Liebow, K Fitzgerald, W Querbes and RJ Desnick. (2014). RNAi-mediated silencing of hepatic Alas1 effectively prevents and treats the induced acute attacks in acute intermittent porphyria mice. Proc Natl Acad Sci U S A 111:7777-7782.

100. Chan A, A Liebow, M Yasuda, L Gan, T Racie, M Maier, S Kuchimanchi, D Foster, S Milstein, et al. (2015). Preclinical development of a subcutaneous ALAS1 RNAi therapeutic for treatment of hepatic porphyrias using circulating RNA quantification. Mol Ther Nucleic Acid 4:e263.

101. Sardh E, P Harper, M Balwani, P Stein, D Rees, J Bloomer, DM Bissell, R Desnick, C Parker, et al. (2018). Phase $1 / 2$, randomized, placebo controlled and open label 
extension studies of Givosiran, an investigational RNA interference (RNAi) therapeutic, in patients with acute intermittent porphyria, http://alnylam.com/wp-content/uploads/ 2018/04/EASL_Ph1-OLE_Capella_FINAL04142018.pdf Accessed on April 23, 2018.

102. Gouya L, JR Bloomer, M Balwani, DM Bissell, DC Rees, U Stölzel, JD Phillips, R Kauppinen, JG Langendonk, et al. (2018). EXPLORE: a prospective, multinational, natural history study of patients with acute hepatic porphyrias (AHPs) with recurrent attacks, http://alnylam.com/wpcontent/uploads/2018/04/EASL_Explore_Capella_FINAL 04142018.pdf Accessed on April 23, 2018.

103. Negrier C, MV Ragni, P Georgiev, T Lissitchkov, H Van Nguyen, K Madigan and KJ Pasi. (2018). Perioperative management in patients with hemophilia receiving Fitusiran, an investigational RNAi therapeutic targeting antithrombin for the treatment of hemophilia, http://alnylam.com/wpcontent/uploads/2017/12/ASH-2017_Negrier_FINAL.pdf Accessed on April 23, 2018.

104. Pasi KJ, S Rangarajan, P Georgiev, T Mant, MD Creagh, T Lissitchkov, D Bevan, S Austin, CR Hay, et al. (2017). Targeting of antithrombin in hemophilia A or B with RNAi therapy. N Engl J Med 377:819-828.

105. Pasi KJ, P Georgiev, T Mant, MD Creagh, T Lissitchkov, D Bevan, S Austin, CR Hay, I Hegemann, et al. (2017). Fitusiran, an investigational RNAi therapeutic targeting antithrombin for the treatment of hemophilia: interim results from a phase 2 extension study in patients with hemophilia $\mathrm{a}$ or $\mathrm{b}$ with and without inhibitors, http:// alnylam.com/wp-content/uploads/2017/07/ISTH-2017_Pasi_ Fitusiran-OLE_CAPELLA-Deck_vFINAL_2017.07.10-1.pdf Accessed on April 23, 2018.

106. Strat AL, CM Ghiciuc, CE Lupussoru and F Mitu. (2016). New class of drugs: therapeutic RNAi inhibition of PCSK9 as a specific LDL-C lowering therapy. Rev Med Chir Soc Med Nat Iasi 120:228-232.

107. Gaudet D. (2016). Novel therapies for severe dyslipidemia originating from human genetics. Curr Opin Lipidol 27: 112-124.

108. Ray KK, U Landmesser, LA Leiter, D Kallend, P Wijngaard, RS Wright and JJP Kastelein. (2017). Impact of a 1- or 2-dose starting regimen of Inclisiran, a novel siRNA inhibitor to PCSK9 on time averaged LDL-C reductions over 1 year, http://clinicaltrialresults.org/Slides/ESC2017/ ORION-1_Ray.pdf Accessed on April 23, 2018.

109. Ray KK, U Landmesser, LA Leiter, D Kallend, P Wijngaard, RS Wright and JJP Kastelein. (2017). LDL-C reduction from 6 to 9 months following single or second injections of inclisiran, a novel siRNA compound, http://alnylam.com/web/ assets/ACC_Ray-401-18_ORION-1_031717.pdf Accessed on April 23, 2018.

110. Liebow A, X Li, T Racie, J Hettinger, BR Bettencourt, N Najafian, P Haslett, K Fitzgerald, RP Holmes, et al. (2017). An investigational RNAi therapeutic targeting glycolate oxidase reduces oxalate production in models of primary hyperoxaluria. J Am Soc Nephrol 28:494-503.

111. Carney EF. (2016). Stones: a novel RNAi therapy for PH1. Nat Rev Nephrol 12:508.

112. Frishberg Y, W van't Hoff, S Hulton, $P$ Haslett, DV Erbe, T McGregor and G Deschênes. (2018). A phase 1/2 trial of lumasiran (ALN-GO1), an investigational RNAi therapeutic for primary hyperoxaluria type 1, http://alnylam.com/wpcontent/uploads/2017/11/Lumasiran-ASN_Capella-Slides_ FINAL11032017.pdf Accessed on April 23, 2018.
113. Alnylam. (2017). Alnylam initiates phase 2 clinical study of cemdisitan (ALN-CC5) in patients with atypical hemolytic-uremic syndrome (aHUS), http://investors .alnylam.com/news-releases/news-release-details/alnylaminitiates-phase-2-clinical-study-cemdisiran-aln-cc5-0 Accessed on April 23, 2018.

114. Rozema DB, DL Lewis, DH Wakefield, SC Wong, JJ Klein, PL Roesch, SL Bertin, TW Reppen, Q Chu, et al. (2007). Dynamic polyconjugates for targeted in vivo delivery of siRNA to hepatocytes. Proc Natl Acad Sci U S A 104:12982-12987.

115. Wong SC, JJ Klein, HL Hamilton, Q Chu, CL Frey, VS Trubetskoy, J Hegge, D Wakefield, DB Rozema and DL Lewis. (2012). Co-injection of a targeted, reversibly masked endosomolytic polymer dramatically improves the efficacy of cholesterol-conjugated small interfering RNAs in vivo. Nucleic Acid Ther 22:380-390.

116. Wooddell CI, DB Rozema, M Hossbach, M John, HL Hamilton, Q Chu, JO Hegge, JJ Klein, DH Wakefield, et al. (2013). Hepatocyte-targeted RNAi therapeutics for the treatment of chronic hepatitis B virus infection. Mol Ther 21:973-985.

117. Yuen MF, HLY Chan, SHK Liu, B Given, T Schluep, J Hamilton, CL Lai, S Locarnini, JY Lau, et al. (2015). ARC-520 produces deep and durable knockdown of viral antigens and DNA in a phase II study in patients with chronic hepatitis B. Hepatology 62:1385A.

118. Yuen M-F, K Liu, BD Given, T Schluep, J Hamilton, C-L Lai, SA Locarnini, K Jackson, J Lau, et al. (2018). RNA interference therapy with ARC-520 injection results in long term off-therapy antigen reduction in treatment naïve patients with chronic HBV, http://ir.arrowheadpharma. com/static-files/5b205068-35a4-41c9-942e-cb5ed100c2b2 Accessed on April 23, 2018.

119. Buchanan J. (2016). Arrowhead stock falls after FDA places hold on clinical trial, https://xconomy.com/wisconsin/2016/ 11/09/arrowhead-stock-falls-after-fda-places-hold-onclinical-trial/ Accessed on April 23, 2018.

120. Wooddell C, R Zhu, H Hamilton, Q Chu, H Sternard, J Schumacher, T Schluep, M Seefeld, Z Li and B Given. (2018). Development of subcutaneously administered RNAi therapeutic ARO-HBV for chronic hepatitis B virus infection. J Hepatol 68:S18-S19.

121. Wooddell C, K Blomenkamp, H Chen, J Griffin, R Zhu, Q Chu, H Hamilton, J Hegge, D Christianson, et al. (2018). AROAAT, a subcutaneous RNAi-based therapeutic for alpha-1 antitrypsin-related liver disease, demonstrates liver exposureresponse and efficacy in preclinical studies. J Hepatol 68:S82.

122. Dicerna. (2018). Corporate Overview, http://investors.dicerna .com/static-files/b0fe07db-a91e-4be5-a422-03d9fd6ab93e Accessed on April 23, 2018.

123. Webb C. (1963). The Graduate. The New American Library, ISBN 0743456459.

$$
\begin{array}{r}
\text { Address correspondence to: } \\
\text { Steven F. Dowdy, PhD } \\
\text { Department of Cellular and Molecular Medicine } \\
\text { University of California San Diego } \\
\text { La Jolla, CA 92093-0686 } \\
\text { E-mail: sdowdy@ucsd.edu }
\end{array}
$$

Received for publication April 25, 2018; accepted after revision April 26, 2018. 